\title{
Identification of promoter elements in the Dolichospermum circinale AWQC131C saxitoxin gene cluster and the experimental analysis of their use for heterologous expression.
}

\section{Paul Michael D'Agostino}

Technische Universitat Munchen

\section{Bakir Al-Sinawi}

University of New South Wales

\section{Rabia Mazmouz}

University of New South Wales

Julia Muenchhoff

University of New South Wales

\section{Brett Anthony Neilan}

University of Newcastle

Michelle Moffitt ( $\square$ m.moffitt@westernsydney.edu.au )

University of Western Sydney https://orcid.org/0000-0001-9872-3175

\section{Research article}

Keywords: cyanobacteria, Dolichospermum circinale, Anabaena circinalis, saxitoxin, paralytic shellfish toxins, regulation, promoter, transcription, heterologous expression

Posted Date: January 15th, 2020

DOI: https://doi.org/10.21203/rs.2.15635/v2

License: (9) This work is licensed under a Creative Commons Attribution 4.0 International License. Read Full License

Version of Record: A version of this preprint was published at BMC Microbiology on February 17th, 2020. See the published version at https://doi.org/10.1186/s12866-020-1720-3. 


\section{Abstract}

Background: Dolichospermum circinale is a filamentous bloom-forming cyanobacterium responsible for biosynthesis of the paralytic shellfish toxins (PST), including saxitoxin. PSTs are neurotoxins and in their purified form are important analytical standards for monitoring the quality of water and seafood and biomedical research tools for studying neuronal sodium channels. More recently, PSTs have been recognised for their utility as local anaesthetics. Characterisation of the transcriptional elements within the saxitoxin ( sxt ) biosynthetic gene cluster (BGC) is a first step towards accessing these molecules for biotechnology. Results: In D. circinale AWQC131C the sxt BGC is transcribed from two bidirectional promoter regions encoding five individual promoters. These promoters were identified experimentally using $5 \square$ RACE and their activity assessed via coupling to a lux reporter system in E. coli and Synechocystis sp. PCC 6803. Transcription of the predicted drug/metabolite transporter (DMT) encoded by sxtPER was found to initiate from two promoters, P sxtPER1 and P sxtPER2 . In E. coli, strong expression of lux from $P$ sxtP , $P$ sxtD and sxtPER1 was observed while expression from $P$ orf 24 and $P$ sxtPER2 was remarkably weaker. In contrast, heterologous expression in Synechocystis sp. PCC6803 showed that expression of lux from P sxtP , P sxtPER, and P orf24 promoters was statistically higher compared to the non-promoter control, while P sxtD showed poor activity under the described conditions. Conclusions: Both of the heterologous hosts investigated in this study exhibited high expression levels from three of the five sxt promoters. These results indicate that the majority of the native sxt promoters appear active in different heterologous hosts, simplifying initial cloning efforts. Therefore, heterologous expression of the sxt BGC in either E. coli or Synechocystis could be a viable first option for producing PSTs for industrial or biomedical purposes.

\section{Background}

Saxitoxin (STX) is a neurotoxin produced by cyanobacteria and dinoflagellates and is a member of the broader group of alkaloids known as the paralytic shellfish toxins (PSTs) [1]. When high concentrations of PSTs are consumed by humans, acute poisoning can lead to death due to respiratory paralysis [2-4]. Therefore, PSTs are needed as analytical standards for the monitoring and protection of commercial seafood and freshwater reservoirs, as well as for use in biomedical research. While the PSTs pose a significant public health risk and economic burden on society during algal bloom events, their scientific and pharmaceutical potential is well known $[3,4]$. Purified PSTs have been a critical tool for researchers investigating neuronal sodium channels, where the toxins specifically block site 1 of voltage-gated sodium channels [5]. Under controlled administration, PSTs are potent anaesthetics, particularly in combination with other local anaesthetics [6, 7]. Further attempts to utilise STX in clinical trials are hindered by its toxicity, but more recent approaches, such as generating liposomal formulations of STX, resulted in blockage of the sciatic nerves in rats with no myotoxic, cytotoxic or neurotoxic effects [8]. It has been postulated that the same delivery could provide effective localised treatment for severe joint pain [9]. Other PSTs, such as the gonyautoxins (GTXs), also have clinical potential and have been utilised for the treatment of anal fissures and chronic tension type headaches [10-12]. 
Obtaining significant quantities of purified PSTs for clinical research or water quality analysis is difficult. Chemical synthesis and biocatalytic synthesis of PSTs is complex, difficult to scale up and may not produce all relevant naturally occurring isoforms Analytical calibration standards are commercially available from the National Research Council Canada (NRC), which are obtained from extractions of PST producing dinoflagellates or contaminated shellfish, and in some instances, semi-synthetic conversions of PSTs are required to obtain or broad array of analogues. Thus, the process is difficult, inefficient and costly [18]. These issues clearly highlight the need for an alternative and reliable method for the production and purification of commercial quantities of PSTs.

Heterologous expression of cyanobacterial biosynthetic gene clusters (BGCs) is not well established, in comparison to heterologous expression of Actinomycete BGCs. Streptomyces expression hosts successfully produced more than 90 Actinomycete NPs, most of which are heterologously expressed using native promoters $E$. coli is a suitable host for the heterologous expression of cyanobacterial pathways based on its fast growth rate as previously demonstrated [21, 22]. Initial studies used native promoters to produce the ribosomal peptides patellamide $A$ and $C$, and the microviridins $[23,24]$. Recently, there has been a focus on the heterologous expression of cyanobacterial natural product biosynthetic gene clusters (BGC) including the lyngbyatoxin (Itx) BGC in E. coli using the tetracycline-inducible Ptet $O$ promoter $[21,25]$. The Itx BGC has been the focus of multiple heterologous expression studies due to its relatively small size. It has also been expressed in the cyanobacterium Anabaena sp. PCC 7120, and $E$. coli GB05-MtaA [26, 27]. While the native Itx promoters were active in Anabaena sp. PCC 7120 and drive the production of lyngbyatoxin $A$, the native promoters were not active in E. coli. The addition of Anabaena sp. PCC 7120 sigma factors to the $E$. coli host also failed to induce expression of lyngbyatoxin $A$, suggesting that the heterologous host was unable to recognise the cyanobacterial ribosome binding sites[28]. Subsequently, titres of lyngbyatoxin A close to the native producer were achieved when using the cyanobacteria Anabaena sp. PCC 7120 as a heterologous host [26]. This highlights that discrepancies between cyanobacterial promoter efficiencies in different host organisms remain poorly understood. Therefore, it is beneficial to test the activity of promoters in heterologous hosts and expression vectors using reporter systems prior to cloning complex biosynthetic pathways for biotechnological applications [29].

For the most part, cyanobacterial transcription machinery is similar to that found in E. coli, with the main difference being the widespread absence of the -35 hexamer in cyanobacteria, which is believed to be replaced by a transcription factor binding site to initiate transcription [30]. In E. coli, $\sigma^{70}$ is able to recognise the majority of promoters while in cyanobacteria, a range of different sigma factors have been identified [31, 32].

The saxitoxin (sxt) BGC has been characterised in six cyanobacterial species from the order Nostocales and one from the order Oscillatoriales [33-37]. Each sxt BGC encodes a 'core' set of enzymes putatively responsible for STX biosynthesis, supplemented with 'tailoring' and 'auxiliary' genes that give rise to PST analogues or perform functions after PST biosynthesis. Information regarding the regulation of transcriptional elements of cyanobacterial secondary metabolite biosynthesis remains limited to the 
microcystin (mcy) and jamaicamide (jam) BGCs [38-43]. However, the regulation of PSTs at the molecular level, including the transcriptional elements of the sxt BGC remains largely unknown $[44,45]$.

Here, we identify the transcription units of the sxt BGC within the cyanobacterium Dolichospermum circinale AWQC131C which enabled the experimental isolation of five promoter regions. We then assessed the reliability of a lux reporter system to assay the activity of uncharacterised cyanobacterial promoters in the heterologous hosts E. coli and Synechocystis sp. PCC 6803 for the first time. Characterisation of these cyanobacterial promoters and determination of their activity in E. coli and Synechocystis, highlights the unpredictability of cyanobacterial promoters of natural product BGCs in heterologous hosts. This study is the first essential phase of understanding the cloning strategy expression of PST biosynthesis, identifying the need for promoter engineering or exchange in future experiments.

\section{Results And Discussion}

\section{Identification of transcriptional units within the sxt biosynthetic gene cluster}

Reverse-transcriptase PCR revealed that the sxt BGC in D. circinale AWQC131C is transcribed as five transcriptional units from two bidirectional promoter regions (Figure 1). All five transcripts appear to be constitutively expressed under standard laboratory conditions, since sxtmRNA was detected across all time points. Operon 1, sxtDV*EABC (* indicates disrupted ORF of $s x t V[34]$ ), spans $7.3 \mathrm{~kb}$, is transcribed in the reverse direction and encodes several proteins predicted to be involved early in PST biosynthesis. Operon 2, sxtPQR, spans $3.5 \mathrm{~kb}$ and is transcribed in the forward direction. The catalytic functions of SxtP, SxtQ and SxtR are unknown, but they are likely to be essential for PST biosynthesis as their presence and organisation is conserved amongst all reported sxt clusters. The third transcriptional unit is monocistronic and encodes SxtPER, a putative permease of the drug/metabolite transporter family of proteins and is transcribed from two promoters, as discussed further below. Operon 4, is transcribed in the forward direction and spans $12.8 \mathrm{~kb}$. Operon 4 encodes a protein of unknown function, Orf 24 that is conserved in most $s x t$ clusters, followed by genes encoding twelve enzymes involved in PST biosynthesis, resulting in the polycistron orf24sxtSTUNGHMIJKLO.

The 3' ends of operons 1-4 were bioinformatically screened for putative Rho-dependent and Rhoindependent transcriptional termination sites using the programs TransTerm and TranstermHP, respectively $[46,47]$. Rho-independent transcription termination sites were identified in the non-coding regions of three out of four sxt mRNA transcripts (Table S1). Rho-dependent or Rho-independent termination sites were not identified in the sequence of mRNA encoding operon 1. 


\section{Transcription start sites and promoter regions of the sxt operons}

The transcriptional start sites (TSSs) of each operon were experimentally identified via 5 ' rapid amplification of cDNA ends (5' RACE) (Table 1; Supporting Information). The upstream region of each TSS was screened for a promoter sequence consistent with conserved binding sequences of group 1, 2 and 3 sigma factors [31]. All promoters identified in this study displayed sequence similarity to the consensus -10 hexamer (Pribnow box) of the prokaryotic RNA polymerase binding site, while there was sporadic presence of the -35 hexamer binding site (Table 3.1). These results suggest that the $s x t$ promoters of $D$. circinale AWQC131C are activated by an RNA polymerase core enzyme in conjunction with a group 1 or group 2 sigma factor [31,48]. For the -10 promoter sequences identified, a search was conducted for an extended -10 binding site and upstream (UP) element. The 5 ' untranslated region (UTR) of each operon was also bioinformatically screened for the presence of consensus ribosomal binding site (RBS) sequences, although previously reported bioinformatics surveys of cyanobacterial genomes were not able to identify the consensus RBS sequence in all genes $[49,50]$. Based on the 5 ' RACE and bioinformatics data, the $D$. circinale AWQC131C sxt BGC includes of a total of five TSSs under standard culture conditions (Figure 2).

Table 1: Characteristics of promoter regions in the sxt biosynthetic gene cluster of $D$. circinale AWQC131C.

\begin{tabular}{|c|c|c|}
\hline Promoter & Promoter sequence $5^{\prime}$ à $3^{\prime}$ & Position $^{\Delta}$ \\
\hline PsxtD & tgtcTTGTGG....(14bp).....GAgTATACTtgactagtA & -32 \\
\hline PsxtP & gtatCTATCA....(12bp).....GTgTATACTagtcaagtA & -34 \\
\hline PsxtPER1 & ttccTTGCAA....(15bp).....AGtTACAATtacatgA & -91 \\
\hline PsxtPER2 & tgagATGACA ....(21bp).....CGaTATATTttgggtG & +94 \\
\hline Porf24 & aaaaTTTCCT....(15bp).....TGcTATAATgaaatcT & -160 \\
\hline E. coli $\sigma^{70}$ consensus & ....TTGACA ....(14bp),....TGnTATAAT.......N & \\
\hline
\end{tabular}

The -10 and -35 hexamers are capitalised and conserved nucleotides are in bold print. $\mathrm{N}$ indicates transcriptional start site (TSS). ${ }^{\Delta}$ Position of promoter relative to TSS.

Operon $1\left(s x t D V^{\star} E A B C\right)$ contains a short $5^{\prime}$ UTR of -32 bp upstream of the translation start site and a promoter $(\mathrm{P} s x t D)$ with high sequence similarity to the $E$. coli $\sigma^{70}-10$ and -35 hexamers. PsxtP initiates the transcription of operon 2, possesses a short $5^{\prime}$ UTR spanning 34 bp and contains both -10 and -35 regions. The transcript initiated by PsxtP also displayed a likely RBS (AAGA) 6 nucleotides upstream of the sxtP translation start site. A conserved -35 sequence was also identified 21 bp upstream of the extended -10 sequence, resulting in an unusually long distance between the two hexamers. Porf 24 has a perfectly conserved -10 consensus sequence, including the extended -10 TGn motif (Table 1). The 5' UTR for orf24 is $160 \mathrm{bp}$ in length. 
Unusually, transcription of the putative transporter, sxtPER, was initiated from two promoters, PsxtPER1 and PsxtPER2. PsxtPER1 is located 91 bp upstream of the annotated TSS of sxtPER (Figure 2) and contains a highly conserved -10 and -35 RNA polymerase binding site. PsxtPER2 is located $94 \mathrm{bp}$ downstream of the translational start site and contains a highly conserved -10 sequence, including the single nucleotide seen in extended -10 promoters as well as a RBS (AAAGAAG). While uncommon, the use of a second TSS to produce two protein isoforms has previously been reported

\section{Promoter activity in E. coli}

The five promoters identified in the D. circinale sxt cluster using 5' RACE, PsxtP, PsxtD, PsxtPER1, PsxtPER2 and Porf24, were amplified by PCR and cloned into the E. coli expression vector, pET28b (Novagen), directly in front of a luciferase reporter (Iux) operon (Figure 3). Expression of luciferase from each of these promoters was measured and compared with negative controls; $p E T 28-I u x$ harbouring a non-promoter region from within the sxtO gene and the pET28-Iux plasmid with no added promoter. Unpaired t-tests showed that all promoters exhibited significant levels of expression (Table S2) when compared to the pET28-Iux negative control. Under the described culture conditions, the heterologous PsxtD, PsxtP, and PsxtPER 1 promoters mediated the highest levels of luciferase expression in $E$. coli (Table S3). There was a statistically significant difference $(p<0.0001)$ between the highest performing promoter PsxtD and all the other promoters, as well as the controls (sxtO and pET28-Iux) (Table S4).

When luciferase expression from each promoter was compared to expression from the non-promoter sxtO fragment control, the strongest promoters (PsxtD, PsxtP, and PsxtPER1) resulted in a 1,000 to 10,000-fold increase in expression (Figure 3B).

The expression of PsxtP is an interesting example of the promoter elements required for heterologous expression of cyanobacterial promoters in E. coli. PsxtP does not seem to have a discernible -35 binding region yet does have a RBS and promoted high expression levels in E. coli. Previous studies have shown that while the distance between the -10 and -35 sequences can effect transcription in cyanobacteria, the -35 hexamer is not always required [59-61]. Thus, the competing preferences between the TSS sequence and position, taken together with other elements of the promoter such as -10 , and -35 sequences, transcription factors, the sequence length between the -10 and -35 regions, and the RBS, highlights the complexity of transcriptional regulation and shows the importance of experimental validation of promoter activation data to further improve bioinformatics databases.

The promoter responsible for the transcription of orf24 and the second promoter of sxtPER, PsXtPER2 were weaker than the other promoters, but still significantly stronger than the controls (Figure 3A).The incorporation of both promoters into the lux expression constructs resulted in a 12-27-fold increase in luciferase expression over sxtO-lux (Figure 3B), and 810-1770-fold increase in luciferase expression over the $\mathrm{pET} 28-\mathrm{Iux}$ control. These results indicate that the promoters are active, albeit weaker than the other three promoters.

\section{The activity of sxt promoters in Synechocystis sp. PCC 6803}


Four sxt promoters were active in Synechocystis sp. PCC 6803 (Figure 4). Unpaired t-tests showed that expression of luciferase from the PsxtP, PsxtPER1, and Porf24 were significantly different to expression in the control strain (Table S6), while expression from PsxtD was not statistically different to the control strain $(P<0.05 ;$ Table S7).

The $s x t D$ promoter regulates the transcription of operon 1 of the $D$. circinale sxt cluster, which carries the core biosynthetic genes, including the polyketide synthase-like enzyme, sxtA. Strains harbouring PsxtD had very low luciferase expression levels that were only 1.3-fold higher than expression levels in the promoter-less control strain. The lack of statistically significant expression from PsxtD indicates the promoter as the only candidate for exchange for heterologous expression of PSTs in Synechocystis sp. PCC 6803.

PsxtP and Porf24 mediated consistent levels of luciferase expression per $\mathrm{OD}_{730}$ throughout the experiment (Figure 4B) PsxtPER1 mediated expression levels that were initially up to three-fold higher than PsxtP, however, the rate of expression decreased over the course of growth. This indicates the majority of toxins could be exported from the cell early in culture and retained in the cell as the culture progresses. This would allow future research to optimise PST extraction at different culture stages, either from the cell free component or the cell mass. Alternatively, since PsxtPER 1 is active at the early growth stages within the heterologous host, it could be a target for repression to limit toxin export and therefore retaining toxin within the cell. This will increase the efficiency of toxin isolation from the cell biomass. [44]

It is known that gene expression levels will have significant impact on the amount of PST molecule produced. Here, the promoters that regulate PST biosynthesis mediated lowered expression of luciferase in Synechocystis sp. PCC 6803 compared to E. coli. The significant decrease in luciferase expression by the cyanobacterial promoters in Synechocystis sp. PCC 6803 has previously been observed in studies of the zinc inducible promoter, Psmt, from Synechococcus sp. PCC7002. Psmt mediated higher levels of protein synthesis and therefore higher levels of ethylene production in E. coli, compared to Synechocystis sp. PCC 6803, which only produced residual levels [62]. Conversely, high expression levels impacted lynbyatoxin biosynthesis in E. coli. Heterologous expression of lyngbyatoxin (ItxA-D) in E. coli was only successful when the strong T7 phage promoter was replaced with the weaker Ptet $O$ promoter [27]. Ptet $_{O}$ has since been exploited for the heterologous expression of multiple cyanobacterial BGCs in E. coli [21, 22, 25]. Subsequent heterologous expression of lyngbyatoxin in Anabaena using non-native promoters was more successful than the E. coli system while expression from the native promoter did not occur in either case [23]. Together, these results suggest that native promoters are recognised differently in heterologous hosts and that while successful transcription of cyanobacterial BGCs in heterologous hosts is important, other factors play a role in the efficiency of the host production of secondary metabolites. This study has identified each of the five native sxt promoters and established through the use of the lux reporter, which of those were not recognised in both E. coli and Synechocystis. 


\section{Conclusion}

PSTs have a range of biomedical applications and thus heterologous expression of the sxt BGC should be explored as a potential tool for the characterisation, manipulation and sustainable production of these compounds. Heterologous expression of cyanobacterial natural product BGCs has had mixed success in the past and further characterisation of cyanobacterial promoters is required for successful expression of complex biosynthetic pathways such as the PST biosynthetic pathway. This study identified five putative sxt promoters in D. circinale AWQC131C and tested their activity in E. coli and Synechocystis sp. PCC 6803. In E. coli, PsxtD, PsxtP and PsxtPER1 promoted high levels of luciferase expression while Porf24 was significantly weaker. Further, if Synechocystis sp. PCC6803 is to be used as a host, the inactive PsxtD should be replaced by host-compatible promoters. Successfully manipulating the sxt BGC within a heterologous host at the transcription level is the first step to efficiently access the PSTs for a range of biotechnological applications.

\section{Methods}

\section{Strains and culture conditions}

D. circinale AWQC131C was maintained in Jaworski's medium (JM) [63] at $24^{\circ} \mathrm{C} \pm 1^{\circ} \mathrm{C}$ and illuminated with $11 \mu \mathrm{mol} \mathrm{m} \mathrm{m}^{-2} \mathrm{~s}^{-1}$ of photons on a 12:12 h light/dark cycle. Synechocystis sp. PCC 6803 was maintained in BG11 medium supplemented with $100 \mathrm{\mu g} \mathrm{mL}^{-1}$ spectinomycin when required, at $30^{\circ} \mathrm{C}$ under constant illumination. Unless otherwise specified, E. coli strains (Table 2) were maintained in Luria broth or on agar plates supplemented with $100 \mathrm{~g} \mathrm{~mL} \mathrm{~m}^{-1}$ ampicillin or $50 \mu \mathrm{g} \mathrm{mL}^{-1}$ kanamycin and grown at $37^{\circ} \mathrm{C}$. 
Table 2: Strains and plasmids

\begin{tabular}{|c|c|c|}
\hline Organism & Purpose & Ref. \\
\hline DH5 $\alpha$ & Expression host of lux reporter assay & Promega \\
\hline TOP 10 & Transformation and propagation of plasmids & Invitrogen \\
\hline $\begin{array}{l}\text { D. } \\
\text { circinale AWQC131C }\end{array}$ & Organism harbouring native $s x t$ cluster & [34] \\
\hline $\begin{array}{l}\text { Synechocystis sp. } \\
\text { PCC } 6803\end{array}$ & Expression host of lux reporter assay & \\
\hline Synechocystis SXL7 & $\Delta p h b C:: \mathrm{P} s x t D-l u x C D A B E-\mathrm{Spec}^{\mathrm{R}}$ & This study \\
\hline Synechocystis SXL8 & $\Delta p h b C:: \mathrm{P} s x t P-l u x C D A B E-\mathrm{Spec}^{\mathrm{R}}$ & This study \\
\hline Synechocystis SXL9 & $\triangle p h b C::$ PsxtPER1-luxCDABE - Spec ${ }^{\mathrm{R}}$ & This study \\
\hline $\begin{array}{l}\text { Synechocystis } \\
\text { SXL10 }\end{array}$ & $\Delta p h b C::$ Porf24-luxCDABE $-\mathrm{Spec}^{\mathrm{R}}$ & This study \\
\hline $\begin{array}{l}\text { Synechocystis } \\
\text { SXL11 }\end{array}$ & $\triangle p h b C::$ lux $C D A B E-\mathrm{Spec}^{\mathrm{R}}$ & This study \\
\hline Plasmids & Purpose & Ref. \\
\hline $\mathrm{pCR}^{\mathrm{TM}} 2.1 \mathrm{TOPO}$ & Cloning and sequencing of $5^{\prime}$ RACE TSS products & Invitrogen \\
\hline pGEM T-Easy & Cloning and sequencing of $5^{\prime}$ RACE TSS products & Promega \\
\hline pET28b & Cloning and expression of $s x t$ promoters & Novagen \\
\hline pSYN_6.3 & $\begin{array}{l}\text { Cloning and transformation of sxt promoters in } \\
\text { Synehcocystis sp. PCC } 6803\end{array}$ & Unpublished \\
\hline pLUX NS II & $\operatorname{lux} C D A B E$ & [64] \\
\hline pSXL1 & pET28b::P sxtD-luxCDABE & This study \\
\hline pSXL2 & pET28b::P sxtP-luxCDABE & This study \\
\hline pSXL3 & pET28b::PsxtPER1-luxCDABE & This study \\
\hline pSXL4 & pET28b::Porf24-IuxCDABE & This study \\
\hline pSXL5 & pET28b::PsxtPER2-luxCDABE & This study \\
\hline pSXL6 & pET28-sxtO-IuxCDABE & This study \\
\hline pET28-lux & pET28-luxCDABE & This study \\
\hline pSXL7 & pSYN_6.3::PsxtD- IuxCDABE & This study \\
\hline pSXL8 & pSYN_6.3::PsxtP- IuxCDABE & This study \\
\hline pSXL9 & pSYN_6.3::PsxtPER1- luxCDABE & This study \\
\hline pSXL10 & pSYN_6.3::Porf24- lux CDABE & This study \\
\hline pSXL11 & pSYN_6.3::IuxCDABE & This study \\
\hline
\end{tabular}

\section{Total RNA extraction, cDNA synthesis and transcriptional analysis}

To extract high quality total RNA, cell pellets were snap-frozen in liquid nitrogen and ground into a fine powder with a mortar and pestle prior to extraction with the RNeasy Plant Mini kit (QIAGEN). Residual genomic (g) DNA was removed from total RNA samples using TURBO DNA-free ${ }^{\text {TM }}$ DNase as described by the manufacturer (Ambion). Removal of contaminating gDNA was confirmed via PCR with the 27F/809R PCR primer set targeting the cyanobacterial 16S rRNA gene [65]. RNA quality was also checked via formaldehyde gel electrophoresis, while gDNA was checked by agarose gel electrophoresis. 
The Superscript ${ }^{\circledR}$ III First Strand synthesis system (Invitrogen) was used to reverse transcribe $1 \mu \mathrm{g}$ of total RNA primed with an antisense gene-specific primer (GSP). Transcriptional units were determined by PCR amplification in a $20 \mu \mathrm{L}$ reaction mixture containing $2.5 \mathrm{mM} \mathrm{MgCl} 2,1 \times \mathrm{PCR}$ buffer (Fisher Biotec, Geneworks), 10 pmol dNTPs (Austral Scientific), 10 pmol of GSP, $1 \mathrm{U}$ of Taq polymerase (Fisher Biotec, Geneworks) and sterile Milli-Q water. Thermal cycling was performed in a Bio- Rad 96-well iCycler (BioRad) and began with an initial denaturation cycle of $95^{\circ} \mathrm{C}$ for $4 \mathrm{~min}$, followed by 35 cycles of DNA denaturation at $95^{\circ} \mathrm{C}$ for $20 \mathrm{~s}$ and primer annealing at $55^{\circ} \mathrm{C}$ for $20 \mathrm{~s}$. DNA strand extension was altered to $1 \mathrm{~min}$ for every $1 \mathrm{~kb}$ of amplified product. A final extension at $72^{\circ} \mathrm{C}$ for $7 \mathrm{~min}$ and a final holding temperature of $4^{\circ} \mathrm{C}$ completed the thermal cycling. Each reaction contained cDNA as the template and two primers (Table S8), which were designed to target an adjacent gene. Amplification was observed if the two adjacent genes were located on the same mRNA transcript. The positive control for each PCR contained gDNA. Two negative control reactions were performed by adding template from a cDNA synthesis reaction, the first omitting reverse transcriptase and the second reaction omitting a nucleic acid template.

\section{Isolation of $D$. circinale AWQC131C sxt biosynthetic gene cluster transcription start sites (TSS) and promoters using 5 ' rapid amplification of cDNA ends (5'RACE)}

To isolate the promoter of each transcriptional unit, TSSs were localised with the FirstChoice ${ }^{\circledR}$ RLM-RACE kit for 5 ' RACE (Ambion) with $10 \mu \mathrm{g}$ total RNA as starting material. The 5' RACE adapter was ligated directly onto RNA followed by reverse transcription cDNA synthesis. First round PCR reactions were performed using a 5 ' outer adapter primer in conjunction with four reverse GSPs at approximately 50100 bp intervals (Figure S1, Table S9). Reactions containing amplified products from the first round PCR became the template for second round nested PCR containing a 5 ' adapter inner primer in conjunction with the same four reverse primers. Amplicons of interest were analysed on a $2 \%(w / v)$ agarose gel and purified using a QIAquick spin gel extraction kit (QIAGEN). Purified PCR products were then cloned into the pGEM-T Easy vector (Promega) and sequenced using an ABI 3730 capillary sequencer at the Ramaciotti Centre for Genomics, UNSW.

\section{Cloning and transformation}

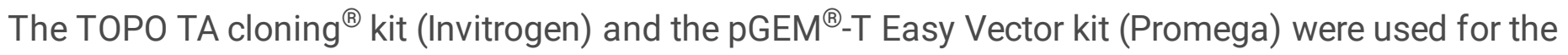
cloning and transformation of $E$. coli (Table 2). Cloning with the TOPO TA cloning ${ }^{\circledR}$ kit involved setting up a ligation reaction containing $4 \mu \mathrm{L}$ of PCR product, $1 \mu \mathrm{L}$ of Invitrogen salt solution $(1.2 \mathrm{M} \mathrm{NaCl}, 0.06 \mathrm{M}$ $\mathrm{MgCl}_{2}$ ) and $10 \mathrm{ng} \mathrm{pCR}{ }^{\circledR}$ 2.1-TOPO ${ }^{\circledR}$ plasmid DNA (Invitrogen). The ligation reaction was incubated for 20 min at room temperature and was then ready for transformation. The PGEM ${ }^{\circledR}$-T Easy vector ligation reaction contained $1 \times$ rapid ligation buffer (Promega), 50 ng of pGEM ${ }^{\circledR}-T$ Easy vector DNA (Promega), 3 Weiss $\mathrm{U}$ of T4 DNA ligase (Promega) and $3 \mu \mathrm{L}$ of PCR product. The ligation reaction was left to incubate overnight at $4^{\circ} \mathrm{C}$ and was then ready for transformation. Positive transformants were selected by blue and white colony screening and the presence of a cloned insert was confirmed by colony PCR using either 
the primer sets M13F and M13R (pCR $\left.{ }^{\circledR} 2.1-T O P O\right)$ or T7F and M13R (pGEM-T Easy). Plasmids containing an insert were then sequenced.

Engineering the sxt promoter-luciferase reporter constructs for expression in E. coli DH5a

Five promoters (PsxtD, PsxtP, PsxtPER1, Porf24, PsxtPER2) and a non-promoter region within the sxtO open reading frame were cloned into the $\mathrm{pET} 28 \mathrm{~b}$ expression vector along with the luciferase reporter (IUxCDABE) operon from Photorhabdus luminescens (Figure S2A). The luciferase operon (IuxCDABE) was amplified from the pLuxNSII plasmid [64] via PCR (denaturation at $98^{\circ} \mathrm{C}$ for $3 \mathrm{~min}$, followed by 30 cycles of denaturation at $98^{\circ} \mathrm{C}$ for $15 \mathrm{~s}$, annealing step at $60^{\circ} \mathrm{C}$ for $20 \mathrm{~s}$, extension at $72^{\circ} \mathrm{C}$ for $30 \mathrm{~s} / \mathrm{kb}$, and a final extension at $72^{\circ} \mathrm{C}$ for $10 \mathrm{~min}$ ), and the PET28b backbone was also PCR amplified to remove the T7 promoter region. All primers were designed using the NEBuilder assembly tool (Table S10). Doublestranded PCR fragments were amplified using the KAPA HiFi Hotstart DNA polymerase (KAPA biosystems). The pET28b backbone, sxt promoter ( $P s x t)$, and lux operon were assembled using the Gibson assembly master mix (NEB) [66], and incubated at $50^{\circ} \mathrm{C}$, for $1 \mathrm{~h}$. The reaction was transformed into chemically competent $E$. coli $\mathrm{DH} 5 \mathrm{a}$ and positive colonies selected as above.

\section{Engineering the sxt promoter-luciferase reporter constructs for expression in Synechocystis sp. PCC 6803}

The Psxt-lux integration vector was engineered through classical restriction/ligation cloning using the restriction enzymes Notl and Kpnl (NEB). The Psxt-lux fragments were amplified from the pET28b-P-lux vectors using the lacl-P-lux_Notl_F, and lacl-P-lux_KpnI_R primers (Table S10). Linear DNA fragments were digested, purified and ligated into the pSYN_6.3 vector (Figure S2B) using T4 DNA ligase at $22^{\circ} \mathrm{C}$ for $1 \mathrm{~h}$, followed by transformation into $\mathrm{E}$. coli $\mathrm{DH} 5 \mathrm{a}$ and colony screening. Plasmid constructs were confirmed by terminal-end sequencing.

The integration of the Psxt-lux fragments into the Synechocystis sp. PCC 6803 genome (Figure S2C) was achieved via the natural competence of the host [67]. Synechocystis sp. PCC 6803 was grown at $30^{\circ} \mathrm{C}$, with shaking at $100 \mathrm{rpm}$, under constant light until exponential phase, and used to inoculate a $50 \mathrm{~mL}$ $B G 11$ medium to an initial $O D_{730}$ of 0.05 . After $\sim 4$ days of photoautotrophic growth, the cells reached an $\mathrm{OD}_{730}$ of 0.5 , and were harvested by centrifugation at $2,750 \mathrm{~g}$ for $5 \mathrm{~min}$. Cells were resuspended in $2 \mathrm{~mL}$ of fresh BG11 medium, divided into $0.5 \mathrm{~mL}$ aliquots $\left(\mathrm{OD}_{730}\right.$ of 2.5), combined with $10 \mu \mathrm{g}$ of DNA and incubated at $30^{\circ} \mathrm{C}$ for $6 \mathrm{~h}$. A sterile Immobilon Transfer membrane (Merk Millipore) was placed on each BG11 agar plate, overlaid with $200 \mu \mathrm{L}$ of the transformation mixture and incubated for $12 \mathrm{~h}$ under constant illumination at $30^{\circ} \mathrm{C}$. Membranes were transferred to BG11 agar plates containing $25 \mu \mathrm{g} \mathrm{mL}^{-1}$ spectinomycin. The plates were incubated for a further two days at $30^{\circ} \mathrm{C}$ under constant illumination, before the membrane was transferred to plates containing $50 \mu \mathrm{g} \mathrm{mL}^{-1}$ spectinomycin and incubated for a further 7-10 days until colonies become visible. Recombinant Synechocystis sp. PCC 6803 colonies were picked and streaked onto BG11 agar plates supplemented with $100 \mu \mathrm{g} \mathrm{mL}^{-1}$ spectinomycin and subcultured a further three times to achieve integration of the cloned reporter fragment and full 
chromosomal segregation. Transformants were confirmed using the PhaCaF and PhaCbR PCR primers (Table S10).

\section{Activity of sxt promoters in E. coli DH5a}

Promoter-luciferase reporter constructs were transformed into E. coli DH5a and grown on M9 minimal medium supplemented with $50 \mu \mathrm{g} \mathrm{mL}^{-1}$ kanamycin at $37^{\circ} \mathrm{C}$ for $24 \mathrm{~h}$. Bioluminescence (RLU) and optical density measurements were measured at one-hour intervals until the $\mathrm{OD}_{600}$ reached 0.8 . A final measurement was taken at $24 \mathrm{~h}$. The strength of each promoter was measured as the highest bioluminescence, normalised to $\mathrm{OD}_{600}$. One-way ANOVA (Graphpad Prism 7) was used to calculate any statistical differences between the promoters. Unpaired t-tests were also used to determine the statistical differences between the strains and the control.

\section{Activity of sxt promoters in Synechocystis sp. PCC 6803}

Promoter-luciferase reporter constructs were transformed into Synechocystis sp. PCC 6803 strains, which were inoculated into BG11 medium supplemented with $100 \mu \mathrm{g} \mathrm{mL}^{-1}$ of spectinomycin and grown at $30^{\circ} \mathrm{C}$ with shaking under constant illumination. Optical density and relative light units (RLU) were measured every $24 \mathrm{~h}$ for $400 \mathrm{~h}$. Promoter strength was measured by determining the highest RLU per $\mathrm{OD}_{730}$. Oneway ANOVA (Graphpad Prism 7) was used to calculate any statistical differences between the promoters. Unpaired t-tests were also used to determine the statistical differences between the strains and the control.

\section{Abbreviations}

5'RACE - rapid amplification of cDNA ends

BGC - biosynthetic gene cluster

GSP - gene specific primer

PST - paralytic shellfish toxins

RBS - ribosomal binding site

TSS - transcriptional start site

UP - upstream

UTR - untranslated region

\section{Declarations}


Not applicable

\section{Consent for publication}

Not applicable

\section{Availability of data and material}

All data generated or analysed during this study are included in this published article and its supplementary information files.

\section{Competing interests}

The authors declare that they have no competing interests

\section{Funding}

This research was funded by the Australian Research Council, grant number DP0880264. PMD and BA were supported by the Australian Postgraduate award. MM was supported by the Western Sydney University Research Development Strategic Initiative Scheme.

\section{Authors' contributions}

PMD, RM, JM, MCM and BAN designed the overall project. PMD performed experimental transcriptional analysis, identification of transcription start site by 5' RACE and bioinformatic analysis. BA and RM designed, cloned and performed promoter activity assays. PMD and BA wrote the manuscript while all authors edited and approved the manuscript.

\section{Acknowledgements}

The authors would like to thank Dr. Adam Jones and Dr. Daniela Ferreira for helpful experimental advice regarding cyanobacterial transcript analysis and identification of the transcription start site. The authors would also like to thank Anna Glöckle and Leanne Pearson for reading and editing the manuscript. The authors would like to thank Professor Carl Johnson for providing the pluxNS II plasmid carrying the luciferase operon, and Professor Dean Price for providing the Synechocystis 6803 strain.

\section{References}

1. Wiese M, D'Agostino PM, Mihali TK, Moffitt MC, Neilan BA: Neurotoxic alkaloids: Saxitoxin and its analogs. Marine Drugs 2010, 8:2185-2211.

2. Etheridge SM: Paralytic shellfish poisoning: Seafood safety and human health perspectives. Toxicon 2010, 56:108-122.

3. Lagos N: Clinical applications of paralytic shellfish poisoning toxins. In Toxins and Biologically Active Compounds from Microalgae. Volume 2. Edited by Rossini GP: CRC Press; 2014: 309-329. 
4. Adams HJ, Blair MR, Jr., Takman BH: The local anesthetic activity of saxitoxin alone and with vasoconstrictor and local anesthetic agents. Archives Internationales de Pharmacodynamie et de Therapie 1976, 224:275-282.

5. Cestèle S, Catterall WA: Molecular mechanisms of neurotoxin action on voltage-gated sodium channels. Biochimie 2000, 82:883-892.

6. Rodriguez-Navarro Alberto J, Lagos N, Lagos M, Braghetto I, Csendes A, Hamilton J, Figueroa C, Truan D, Garcia C, Rojas A, et al: Neosaxitoxin as a local anesthetic preliminary observations from a first human trial. Anesthesiology 2007, 106:339-345.

7. Barnet CS, Tse JY, Kohane DS: Site 1 sodium channel blockers prolong the duration of sciatic nerve blockade from tricyclic antidepressants. Pain 2004, 110:432-438.

8. Epstein-Barash H, Shichor I, Kwon AH, Hall S, Lawlor MW, Langer R, Kohane DS: Prolonged duration local anesthesia with minimal toxicity. Proceedings of the National Academy of Sciences of the United States of America 2009, 106:7125-7130.

9. Chorny M, Levy RJ: Site-specific analgesia with sustained release liposomes. Proceedings of the National Academy of Sciences of the United States of America 2009, 106:6891-6892.

10. Garrido R, Lagos N, Lattes K, Abedrapo M, Bocic G, Cuneo A, Chiong H, Jensen C, Azolas R, Henriquez A, Garcia C: Gonyautoxin: New treatment for healing acute and chronic anal fissures. Diseases of the Colon \& Rectum 2005, 48:335-343.

11. Garrido R, Lagos N, Lattes K, Azolas CG, Bocic G, Cuneo A, Chiong H, Jensen C, Henriquez Al, Fernandez $\mathrm{C}$ : The gonyautoxin 2/3 epimers reduces anal tone when injected in the anal sphincter of healthy adults. Biological Research 2004, 37:395-403.

12. Lattes K, Venegas P, Lagos N, Lagos M, Pedraza L, Rodriguez-Navarro AJ, Garcia C: Local infiltration of gonyautoxin is safe and effective in treatment of chronic tension-type headache. Neurological Research 2009, 31:228-233.

13. Walker JR, Merit JE, Thomas-Tran R, Tang DTY, Du Bois J: Divergent Synthesis of Natural Derivatives of (+)-Saxitoxin Including 11-Saxitoxinethanoic Acid. Angewandte Chemie International Edition 2019, 58:1689-1693.

14. Chun SW, Narayan ARH: Biocatalytic synthesis of a-amino ketones. Synlett : accounts and rapid communications in synthetic organic chemistry 2019, 30:1269-1274.

15. Watanabe R, Suzuki T, Oshima Y: Preparation of calibration standards of N1-H paralytic shellfish toxin analogues by large-scale culture of cyanobacterium Anabaena circinalis (TA04). Marine drugs 2011, 9:466-477.

16. Quilliam M: Certified reference materials for marine toxins. 2006: 139-144.

17. Laycock MV, Thibault P, Ayer SW, Walter JA: Isolation and purification procedures for the preparation of paralytic shellfish poisoning toxin standards. Natural Toxins 1994, 2:175-183.

18. Thomas K, Blay P, Burton IW, Cembella A, Craft C, Crain S, Hardstaff WR, Laycock M, LeBlanc D, LeBlanc P: Certified reference materials for marine algal toxins. In Harmful Algae 2002: Florida Fish 
and Wildlife Conservation Commission and Intergovernmental Oceanographic Commission of UNESCO. Edited by Steidinger KA, Landsberg JH, Tomas CR, Vargo GA2004: 216-218.

19. Nah H-J, Pyeon H-R, Kang S-H, Choi S-S, Kim E-S: Cloning and Heterologous Expression of a Largesized Natural Product Biosynthetic Gene Cluster in Streptomyces Species. Frontiers in microbiology 2017, 8:394-394.

20. Zhang JJ, Tang X, Moore BS: Genetic platforms for heterologous expression of microbial natural products. Natural Product Reports 2019, 36:1313-1332.

21. Liu T, Mazmouz R, Ongley SE, Chau R, Pickford R, Woodhouse JN, Neilan BA: Directing the Heterologous Production of Specific Cyanobacterial Toxin Variants. ACS Chemical Biology 2017, 12:2021-2029.

22. D'Agostino PM, Gulder TAM: Direct pathway cloning combined with sequence- and ligationindependent cloning for fast biosynthetic gene cluster refactoring and heterologous expression. ACS Synthetic Biology 2018, 7:1702-1708.

23. Long PF, Dunlap WC, Battershill CN, Jaspars M: Shotgun cloning and heterologous expression of the patellamide gene cluster as a strategy to achieving sustained metabolite production. ChemBioChem 2005, 6:1760-1765.

24. Ziemert N, Ishida K, Liaimer A, Hertweck C, Dittmann E: Ribosomal synthesis of tricyclic depsipeptides in bloom-forming cyanobacteria. Angewandte Chemie International Edition 2008, 47:7756-7759.

25. Greunke C, Duell ER, D’Agostino PM, Glöckle A, Lamm K, Gulder TAM: Direct pathway cloning (DiPaC) to unlock natural product biosynthetic potential. Metabolic Engineering 2018, 47:334-345.

26. Videau P, Wells KN, Singh AJ, Gerwick WH, Philmus B: Assessment of Anabaena sp. strain PCC 7120 as a heterologous expression host for cyanobacterial natural products: Production of lyngbyatoxin $A$. ACS Synthetic Biology 2016.

27. Ongley SE, Bian X, Zhang Y, Chau R, Gerwick WH, Müller R, Neilan BA: High-titer heterologous production in $E$. coli of lyngbyatoxin, a protein kinase $C$ activator from an uncultured marine cyanobacterium. ACS Chemical Biology 2013, 8:1888-1893.

28. Wells KN, Nelson D, Eiting JE, Videau P, Philmus B: The influence of sigma factors and ribosomal recognition elements on heterologous expression of cyanobacterial gene clusters in Escherichia coli. FEMS Microbiology Letters 2018, 365.

29. Huang $\mathrm{H}-\mathrm{H}$, Camsund D, Lindblad P, Heidorn T: Design and characterization of molecular tools for a synthetic biology approach towards developing cyanobacterial biotechnology. Nucleic Acids Research 2010, 38:2577-2593.

30. Curtis SE, Martin JA: The transcription apparatus and the regulation of transcription initiation. In The Molecular Biology of Cyanobacteria. Volume 1. Edited by Bryant DA. Dordrecht: Kluwer Academic Publishers; 1994: 613-639.[Advances in Photosynthesis and Respiration].

31. Imamura S, Asayama m: Sigma factors for cyanobacterial transcription. Gene Regulation and Systems Biology 2009, 3:65-87. 
32. Imamura S, Yoshihara S, Nakano S, Shiozaki N, Yamada A, Tanaka K, Takahashi H, Asayama M, Shirai M: Purification, characterization, and gene expression of all sigma factors of RNA polymerase in a cyanobacterium. Journal of Molecular Biology 2003, 325:857-872.

33. Kellmann R, Mihali TK, Jeon YJ, Pickford R, Pomati F, Neilan BA: Biosynthetic intermediate analysis and functional homology reveal a saxitoxin gene cluster in cyanobacteria. Applied and Environmental Microbiology 2008, 74:4044-4053.

34. Mihali TK, Kellmann R, Neilan BA: Characterisation of the paralytic shellfish toxin biosynthesis gene clusters in Anabaena circinalis AWQC131C and Aphanizomenon sp. NH-5. BMC Biochemistry 2009, $10: 8$.

35. Stucken K, John U, Cembella A, Murillo AA, Soto-Liebe K, Fuentes-Valdés JJ, Friedel M, Plominsky AM, Vásquez M, Glöckner G: The Smallest Known Genomes of Multicellular and Toxic Cyanobacteria: Comparison, Minimal Gene Sets for Linked Traits and the Evolutionary Implications. PLOS ONE 2010, 5:e9235.

36. Mihali TK, Carmichael WW, Neilan BA: A putative gene cluster from a Lyngbya wollei bloom that encodes paralytic shellfish toxin biosynthesis. PLOS ONE 2011, 6:e14657.

37. Cullen A, D'Agostino PM, Mazmouz R, Pickford R, Wood SA, Neilan BA: Insertions within the saxitoxin biosynthetic gene cluster result in differential toxin profiles. ACS Chemical Biology 2018, 13:31073114.

38. Martin-Luna B, Sevilla E, Hernandez JA, Bes MT, Fillat MF, Peleato ML: Fur from Microcystis aeruginosa binds in vitro promoter regions of the microcystin biosynthesis gene cluster. Phytochemistry 2006, 67:876-881.

39. Ginn HP, Pearson LA, Neilan BA: NtcA from Microcystis aeruginosa PCC 7806 is autoregulatory and binds to the microcystin promoter. Applied and Environmental Microbiology 2010, 76:4362-4368.

40. Neilan BA, Pearson LA, Muenchhoff J, Moffitt MC, Dittmann E: Environmental conditions that influence toxin biosynthesis in cyanobacteria. Environmental Microbiology 2012, 15:1239-1253.

41. Kaebernick M, Neilan BA, Börner T, Dittmann E: Light and the transcriptional response of the microcystin biosynthesis gene cluster. Applied and Environmental Microbiology 2000, 66:3387-3392.

42. Kaebernick M, Dittmann E, Börner T, Neilan BA: Multiple alternate transcripts direct the biosynthesis of microcystin, a cyanobacterial nonribosomal peptide. Applied and Environmental Microbiology 2002, 68:449-455.

43. Jones A, Gerwick L, Gonzalez D, Dorrestein P, Gerwick W: Transcriptional analysis of the jamaicamide gene cluster from the marine cyanobacterium Lyngbya majuscula and identification of possible regulatory proteins. BMC Microbiology 2009, 9:247.

44. Ongley SE, Pengelly JJL, Neilan BA: Elevated Na+ and pH influence the production and transport of saxitoxin in the cyanobacteria Anabaena circinalis AWQC131C and Cylindrospermopsis raciborskii T3. Environmental Microbiology 2016, 18:427-438.

45. D'Agostino PM, Song X, Neilan BA, Moffitt MC: Proteogenomics of a saxitoxin-producing and nontoxic strain of Anabaena circinalis (cyanobacteria) in response to extracellular $\mathrm{NaCl}$ and phosphate 
depletion. Environmental Microbiology 2016, 18:461-476.

46. Jacobs GH, Chen A, Stevens SG, Stockwell PA, Black MA, Tate WP, Brown CM: Transterm: A database to aid the analysis of regulatory sequences in mRNAs. Nucleic Acids Research 2009, 37:D72-D76.

47. Kingsford C, Ayanbule K, Salzberg S: Rapid, accurate, computational discovery of Rho-independent transcription terminators illuminates their relationship to DNA uptake. Genome Biology 2007, 8:R22.

48. Camsund D, Lindblad P: Engineered transcriptional systems for cyanobacterial biotechnology. Frontiers in Bioengineering and Biotechnology 2014, 2.

49. Omotajo $\mathrm{D}$, Tate $\mathrm{T}$, Cho $\mathrm{H}$, Choudhary M: Distribution and diversity of ribosome binding sites in prokaryotic genomes. BMC genomics 2015, 16:604-604.

50. Mutsuda M, Sugiura M: Translation initiation of cyanobacterial rbcS mRNAs requires the 38-kDa ribosomal protein $\mathbf{S 1}$ but not the shine-dalgarno sequence. Journal of Biological Chemistry 2006, 281:38314-38321.

51. Meydan S, Vázquez-Laslop N, Mankin AS: Genes within genes in bacterial genomes. Microbiology Spectrum 2018, 6.

52. Hwang J, Manuvakhova M, Tai PC: Characterization of in-frame proteins encoded by $c v a A$, an essential gene in the colicin V secretion system: CvaA* stabilizes CvaA to enhance secretion. Journal of Bacteriology 1997, 179:689-696.

53. Tate CG, Muiry JA, Henderson PJ: Mapping, cloning, expression, and sequencing of the rhaT gene, which encodes a novel L-rhamnose-H+ transport protein in Salmonella typhimurium and Escherichia coli. Journal of Biological Chemistry 1992, 267:6923-6932.

54. Kim D, Hong JS-J, Qiu Y, Nagarajan H, Seo J-H, Cho B-K, Tsai S-F, Palsson BØ: Comparative analysis of regulatory elements between Escherichia coli and Klebsiella pneumoniae by genome-wide transcription start site profiling. PLoS Genetics 2012, 8:e1002867.

55. Mitschke J, Georg J, Scholz I, Sharma CM, Dienst D, Bantscheff J, Voß B, Steglich C, Wilde A, Vogel J, Hess WR: An experimentally anchored map of transcriptional start sites in the model cyanobacterium Synechocystis sp. PCC6803. Proceedings of the National Academy of Sciences 2011, 108:21242129.

56. Walker KA, Osuna R: Factors Affecting Start Site Selection at the Escherichia coli fis Promoter. Journal of Bacteriology 2002, 184:4783-4791.

57. Liu J, Turnbough CL: Effects of transcriptional start site sequence and position on nucleotidesensitive selection of alternative start sites at the pyrC promoter in Escherichia coli. Journal of Bacteriology 1994, 176:2938-2945.

58. Han X, Turnbough CL: Transcription start site sequence and spacing between the -10 region and the start site affect reiterative transcription-mediated regulation of gene expression in Escherichia coli. Journal of Bacteriology 2014, 196:2912-2920.

59. Dutheil J, Saenkham P, Sakr S, Leplat C, Ortega-Ramos M, Bottin H, Cournac L, Cassier-Chauvat C, Chauvat F: The AbrB2 autorepressor, expressed from an atypical promoter, represses the 
hydrogenase operon to regulate hydrogen production in Synechocystis strain PCC6803. Journal of Bacteriology 2012, 194:5423-5433.

60. Subudhi S, Kurdrid P, Hongsthong A, Sirijuntarut M, Cheevadhanarak S, Tanticharoen M: Isolation and functional characterization of Spirulina D6D gene promoter: Role of a putative GntR transcription factor in transcriptional regulation of D6D gene expression. Biochemical and Biophysical Research Communications 2008, 365:643-649.

61. Shibato J, Agrawal G, Kato H, Asayama M, Shirai M: The 5'-upstream cis-acting sequences of a cyanobacterial $p s b A$ gene: Analysis of their roles in basal, light-dependent and circadian transcription. Molecular Genetics and Genomics 2002, 267:684-694.

62. Guerrero F, Carbonell V, Cossu M, Correddu D, Jones PR: Ethylene synthesis and regulated expression of recombinant protein in Synechocystis sp. PCC 6803. PLOS ONE 2012, 7:e50470.

63. Thompson AS, Rhodes JC, Pettman I: Culture collection of algae and protozoa: Catalogue of strains. Natural Environment Research Council. 5 edn. Ambleside, United Kingdom: Freshwater Biological Association; 1988.

64. Woelfle MA, Xu Y, Qin X, Johnson CH: Circadian rhythms of superhelical status of DNA in cyanobacteria. Proceedings of the National Academy of Sciences 2007, 104:18819-18824.

65. Jungblut A, Hawes I, Mountfort D, Hitzfeld B, Dietrich DR, Burns BP, Neilan BA: Diversity within cyanobacterial mat communities in variable salinity meltwater ponds of McMurdo Ice Shelf, Antarctica. Environmental Microbiology 2005, 7:519-529.

66. Gibson DG, Young L, Chuang R-Y, Venter JC, Hutchison CA, Smith HO: Enzymatic assembly of DNA molecules up to several hundred kilobases. Nat Meth 2009, 6:343-345.

67. Eaton-Rye JJ: Construction of gene interruptions and gene deletions in the cyanobacterium Synechocystis sp. strain PCC 6803. In Photosynthesis Research Protocols. Edited by Carpentier R. Totowa, NJ: Humana Press; 2011: 295-312.

\section{Figures}




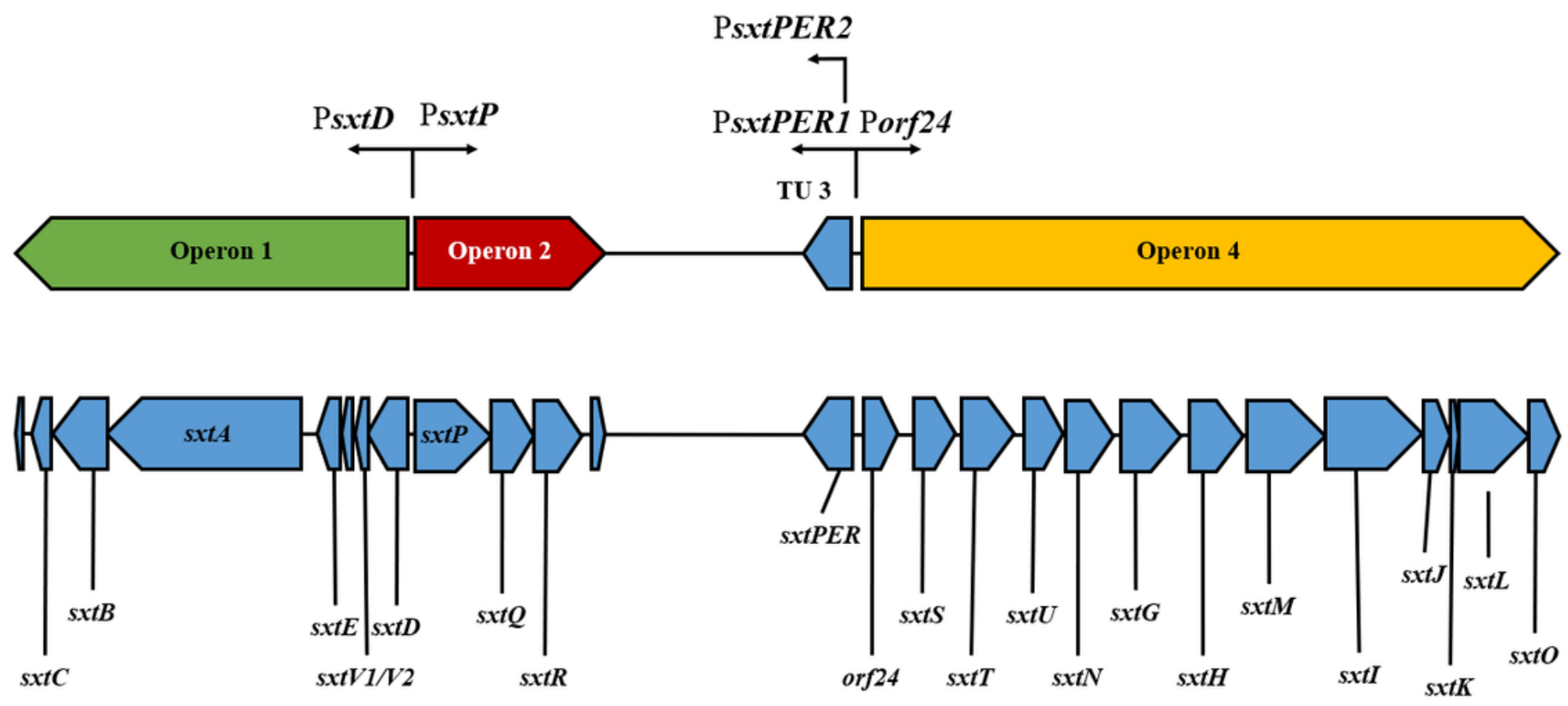

Figure 1

Transcriptional analysis of the D. circinale AWQC131C sxt cluster. Genes required for PST biosynthesis are transcribed by a minimum of four mRNA transcripts from two bi-directional promoter regions encoding five promoters; PsxtD (operon 1), PsxtP (operon 2), PsxtPER1 (TU 3), PsxtPER2 (TU 3) and Porf24 (operon 4). Direction of transcription is indicated by black arrows. 


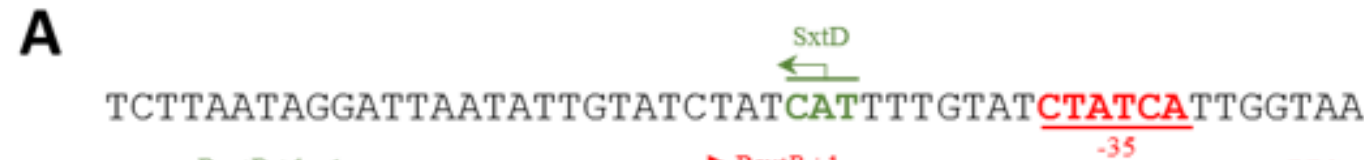
PsxiD $+1 \longleftarrow$

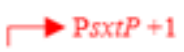

$-35$ CCTTAGTGTATACTAGTCAAGTATACTCTTATTAACAAATTTCCACAAGA

$-35$

CAACTCATGAAAAGGCTCAGTTCGTTAGTTCTAGCAGCACTCTTGTTTGT $\underset{\text { SxtP }}{\longrightarrow}$

B

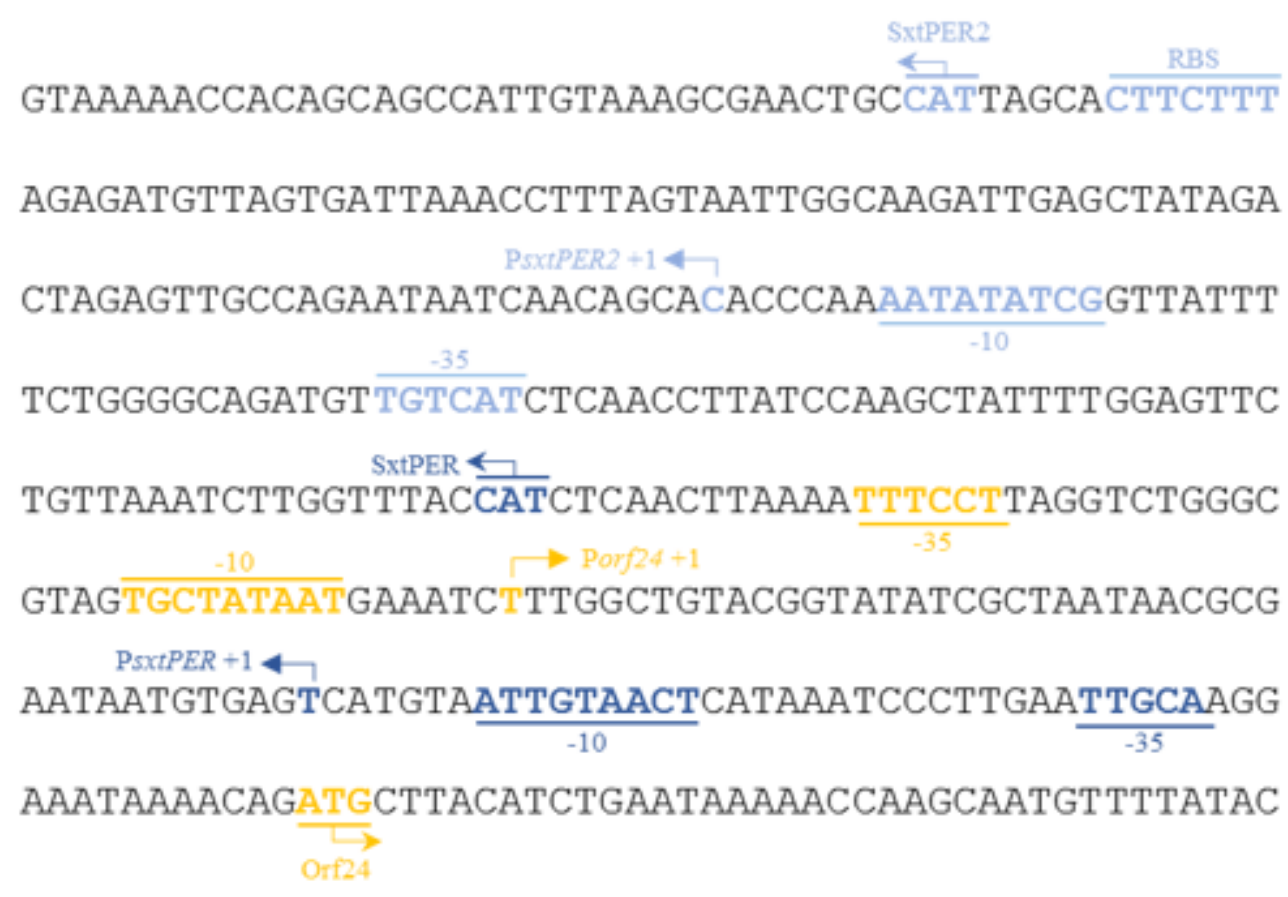

\section{Figure 2}

Sequence analysis of the five promoters present within the D. circinale AWQC131C sxt biosynthetic gene cluster. Individual promoters include, PsxtD (green), PsxtP (red), PsxtPER2 (light blue), PsxtPER1 (dark blue) and Porf24 (yellow). Transcriptional elements including promoters, TSS, translation start site, and RBS are shown for the five sxt promoters. 
A

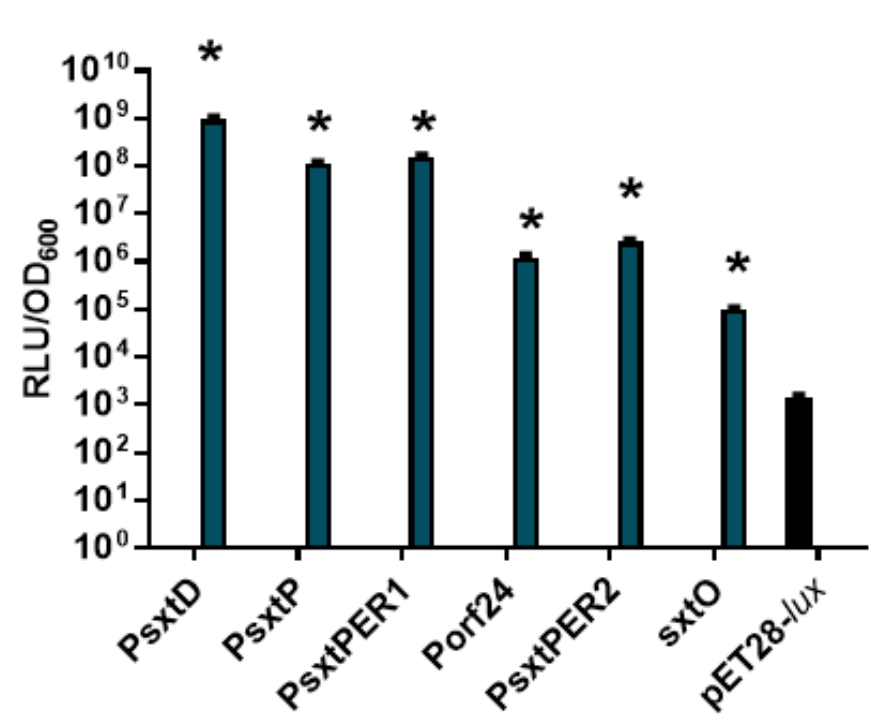

B

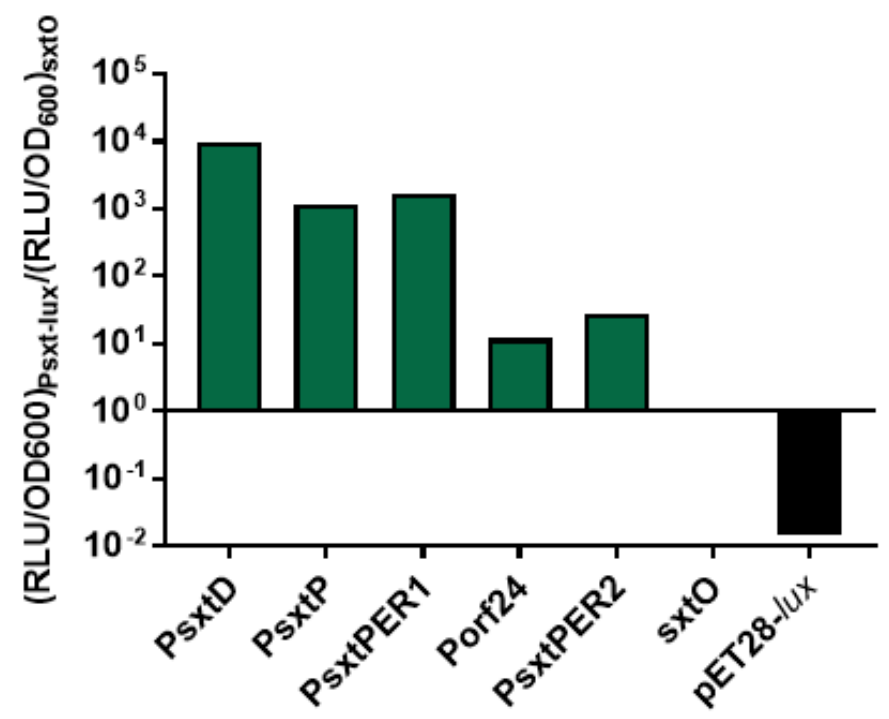

Figure 3

Heterologous expression from of luciferase from sxt promoters in E. coli DH5a. (A) Luciferase expression was normalised to the optical density, OD600. The activity of five promoters was tested: PsxtD, PsxtP, PsxtPER1, Porf24, and PsxtPER2. An intergenic sxtO sequence was used as the non-promoter control. The highest RLU/OD for the E. coli DH5a strains expressing luxCDABE. (*) Denotes statistically significant differences $(p<0.0001)$ between the E. coli DH5a strains and the pET28-lux control in unpaired t-tests. (B) Expression relative to the sxtO-lux control. The mean normalised luciferase expression for each promoter was divided by the mean normalised expression of sxt0. The three strongest promoters (PsxtD, PsxtP, PsxtPER) exhibit between 1000-9500 fold higher levels of luciferase expression over the sxtO control. The Porf24 and PsxtPER2 promoters had only a 12 and 27 -fold increase in expression, respectively. 
A

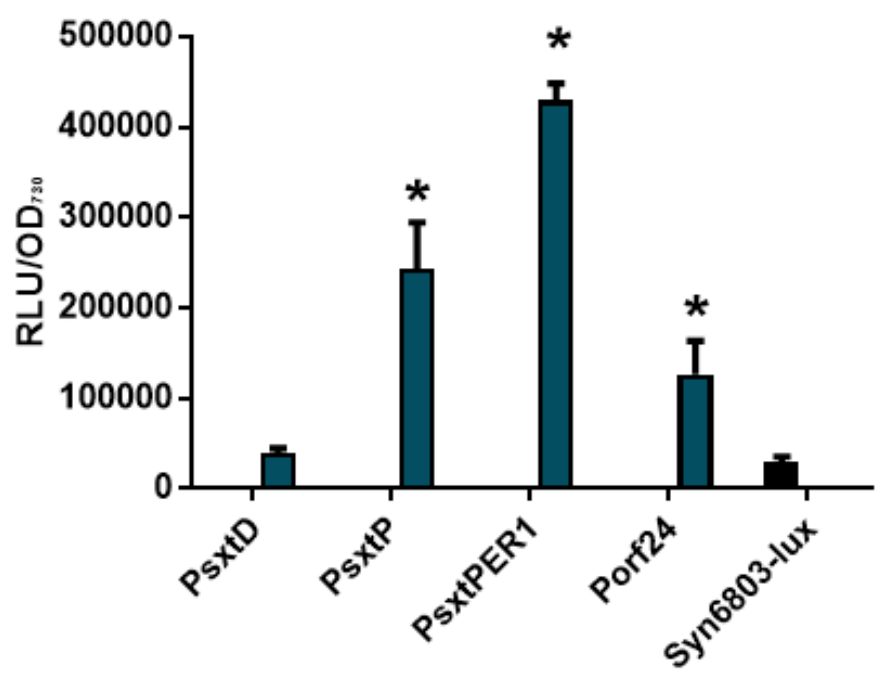

B

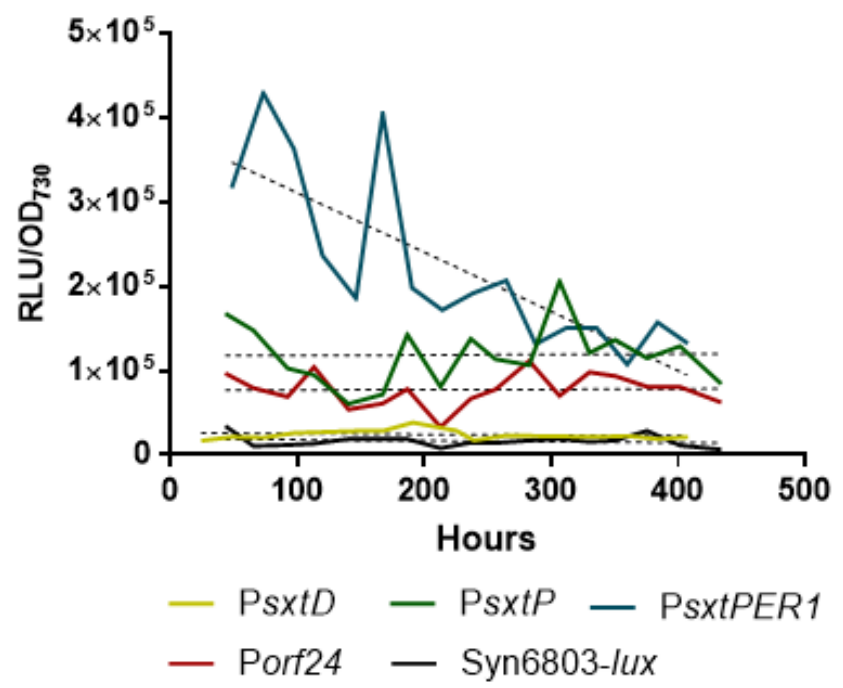

Figure 4

Heterologous expression of luciferase from sxt promoters in Synechocystis sp. PCC 6803. (A) Luciferase expression from the four main sxt promoters in Synechocystis sp. PCC 6803. The highest RLU/OD for the Synechocystis sp.PCC 6803 strains expressing luxCDABE. (*) Denotes statistically significant differences $(p<0.05)$ between the Synechocystis sp. PCC 6803 strains and the Synechcocystis sp. PCC 6803-lux control in unpaired t-tests. (B) Normalised luciferase expression over time in Synechocystis PCC6803. RLU/OD730 over $400 \mathrm{~h}$ of growth. PsxtD normalised expression was low. PsxtP and Porf24 had consistent levels of luciferase expression. PsxtPER showed high initial expression that declined over growth to expression reach levels similar to PsxtP and Porf24.

\section{Supplementary Files}

This is a list of supplementary files associated with this preprint. Click to download.

- 191224aSupportinginfofinalPD.docx 